ORIGINAL ARTICLE

\title{
Outcome of Chronic Subdural Hematoma Treated with Single Burr Hole Under Local Anesthesia
}

\author{
Abebe Mersha ${ }^{1}$, Sahlu Abat ${ }^{1}$, Tsegaye Temesgen ${ }^{1}$, Abebe Nebyou ${ }^{1 *}$
}

\footnotetext{
OPEN ACCESS

Citation: Abebe Mersha,Sahlu Abat, Tsegaye Temesgen,Abebe Nebyou. Outcome of Chronic Subdural Hematoma Treated with Single Burr Hole Under Local Anesthesia. Ethiop J Health Sci.2030;30(1):101.doi:http:// dx.doi.org/ 10.4314/ejhs.v30i1.13

Received: August 06, 2019

Accepted: September 27, 2019

Published: January 1, 2020

Copyright: (C) 2019 Abebe Nebyou, et al.

This is an open access article distributed under the terms of the Creative Commons Attribution License, which permits unrestricted use, distribution, and reproduction in any medium, provided the original author and source are credited. Funding: Nil

Competing Interests: The authors declare that this manuscript was approved by all authors in its form and that no competing interest exists.

Affiliation and Correspondence:

${ }^{1}$ Addis Abeba University, Collage of

health science, School of medicine,

Department of surgery

*Email:nebyouss@yahoo.com
}

\section{ABSTRACT}

BACKGROUND: Chronic subdural hematoma (CSDH) is a common condition encountered in daily neurosurgical practice usually affecting the elderly population. Various surgical procedures have been used for the evacuation of hematoma in patients with CSDH. The objective of this paper was to study the postoperative outcome of patients who were operated for CSDH and to describe the easy, safest and effective procedure that can be performed at primary level hospitals.

METHODS: Institutional based cross-sectional retrospective study was conducted among patients operated for CSDH from January 1, 2012 to December 31, 2015 at Teklehaymanot General Hospital, a private hospital in Addis Ababa, Ethiopia. Descriptive statistics, using SPSS version 20, was used to determine the postoperative outcomes including hospital stay, complications and recurrence rate.

RESULTS: Of the 195 charts reviewed, $70.3 \%$ were of males, with M: $F$ ratio of 2.4:1. 68.2\% of patients being above the age of 55 years with a mean age at presentation of 57.63. The most common presenting symptom was headache followed by extremity weakness. The diagnosis of CSDH was made with either head CT scan or MRI. Forty one percent of patients had a left side hematoma and 48(24.6\%) patients had bilateral CSDH. All patients were operated with a single burr hole evacuation under local anesthesia and postoperative subdural closed system drainage by a single neurosurgeon. The mean hospital stay was $3.68 \pm 2.6$ days. The postoperative outcome was assessed using the Glasgow Outcome Score, and $95.9 \%$ of the patients reported good recovery. Thirteen (6.6\%) patients were operated twice for recurrence, and there were four deaths.

CONCLUSIONS: Single burr hole craniostomy is an easy, safe and effective technique for the treatment of CSDH.

KEYWORDS: Subdural Hematoma, Single Burr Hole, Glasgow Outcome Score, Recollection

\section{INTRODUCTION}

Chronic subdural hematoma (CSDH) is defined as a cystic unclotted hematoma within the outer and inner membranes in the subdural space (1). It is a common condition encountered in daily neurosurgical 
practice usually affecting the elderly population $(2,3,4)$. SDH that develops from 3 days to 3 weeks after head injury is called sub-acute, and those that appear later than 3 weeks after injury are called chronic $(2,3)$. CSDH is formed gradually by the hemorrhage from parasagittal veins following a head trauma. In complicated cases, such as patients with chronic alcoholism, epilepsy, hematological disorders, cerebral atrophy, and under anticoagulant therapy, a minor head trauma may cause CSDH. The major symptoms of $\mathrm{CSDH}$ are a headache, unconsciousness, confusion, and neurological deficits (contralateral motor deficits) (2).

Surgical evacuation of the hematoma is indicated when the CSDH causes symptoms such as focal deficit, mental status changes and/or body weakness, when the volume is significant with the thickness of the hematoma becomes $1 \mathrm{~cm}$ or more, or if it is progressively increasing in size on serial imaging (CT or MRI) (4). Various surgical procedures have been used for the evacuation of hematoma in patients with CSDH. These include: (a) placing two burr holes and irrigating check with tepid saline until the fluid runs clear; (b) single large burr hole with irrigation and aspiration; (c) single burr hole drainage with placement of subdural drain; (d) twist drill craniostomy which has higher recurrence rate, and (e) formal craniotomy with excision of subdural membrane (membranectomy) (3). These procedures can be performed under general or local, anesthesia depending on the protocol of the particular hospital or the preference of the individual surgeon $(3,4,5)$.

Surgical treatment for CSDH is one of the commonest procedures which can be performed safely by a neurosurgeon, general surgeons or residents of both neurosurgery and general surgery. It can be performed under local anesthesia, under monitored anesthesia care (6) or under general anesthesia. Although CSDH is one of the most commonly encountered conditions in neurosurgery, no consensus yet exists regarding the optimal surgical technique to treat the CSDH (7). Describing the safest, easy and effective procedure that can be performed by general and neurosurgery residents and surgeons at the primary level hospitals is very important for developing countries, like Ethiopia, where neurosurgeons and neurosurgical centers are scarce.
Therefore, the aim of this study was to investigate the postoperative outcome of patients who were operated for CSDH by a single neurosurgeon, at a single institution with all patients treated by a single operative procedure.

\section{METHODS AND MATERIALS}

Study design and period: Institutional based crosssectional retrospective study (of a case series) was conducted among patients operated for symptomatic chronic subdural hematoma by a single neurosurgeon and a single operative procedure during a four year period from January 1, 2012 to December 31, 2015 at Teklehaymanot General Hospital, a private hospital in Addis Ababa, Ethiopia. Nonprobability purposive sampling method was used, and all operated cases with complete chart and who had at least four months out patient follow up were included. Of the 203 operated patients registered on the surgical log book, 195 were included in this study with a retrieval rate of $96.0 \%$. Eight cases were excluded as the charts of the two patients could not be retrieved from the archive, and the rest six patients did not have complete four months' follow-up data in their charts.

The procedure: After a detailed history, and general and neurological examination, the diagnosis of CSDH was confirmed with either CT scan or MRI of the brain in all of our patients. The patient was placed on supine position on the operating table and the selected side of the head was prepared by shaving the hair. ECG, pulse oxymetery as well as blood pressure monitors were applied, and 100\% oxygen via nasal catheter was administered. The surgical site was cleaned using alcohol and povidone antiseptic solutions and draped in the usual manner. After a single dose of prophylactic intravenous ceftriaxone $1 \mathrm{gm}$ was given, a $2 \%$ lidocaine with adrenaline of $10 \mathrm{cc}$ was injected to the scalp incision site. IV sedatives, usually valium and pethidine, were added for some patients who were restless and uncooperative from alteration of mentation.

After checking for pain, a four to six centimeter linear scalp incision was made over the parietal prominence and the skull bone was exposed. A single burr hole was then made using Hudson brace hand drill, the dura was opened with a cruciate

DOI: http://dx.doi.org/10.4314/ejhs.v30i1.13 
incision and cauterized. On opening the dura, usually hemolyzed dark blood comes out under pressure and evacuated by inserting soft flexible number 10 pediatric feeding tube. After complete evacuation of the hematoma, the subdural space was irrigated with warm normal saline until clear irrigant is returned. Finally, the subdural space was refilled with normal saline to displace the air out of the subdural space. Leaving the feeding tube drain in the subdural space through a separate scalp incision. Then, the scalp was closed with interrupted silk stich. The drain was connected to closed system collecting bag which is usually a sterile urine bag.

Postoperatively, the patient was kept on supine without pillow for 48 hours with the collecting draining bag by the bed side. After the drainage was complete, i.e. when there was no further fluid coming out and accumulated to the draining bag or nil to the amount of 10 to $20 \mathrm{cc}$, usually 12 to 24 hours, the drainage tube was removed, but the patient remained in supine position for 48 hours after the procedure. Most patients were discharged from hospital on the second or third postoperative day and stiches were removed on the $10^{\text {th }}$ postoperative, day. In our hospital, all of our patients have their first postoperative follow-up on the $10^{\text {th }}$ postoperative day, with the second visit a month later and finally three months after the last visit, at which time they are released from follow-up after strictly advising them on danger signs and to come back should any new symptom develops. The clinical status of the patients at the last postoperative follow-up was assessed using the Glasgow Outcome Score (Table $1)$.

Table 1: Glasgow Outcome Score

\begin{tabular}{ll}
\hline SCORE & DESCRIPTION \\
\hline $\mathbf{5}$ & $\begin{array}{l}\text { Good recovery, resumption of normal life } \\
\text { Moderate disability, disabled but } \\
\text { independent }\end{array}$ \\
$\mathbf{4}$ & $\begin{array}{l}\text { Severe disability, dependent for daily } \\
\text { support }\end{array}$ \\
$\mathbf{3}$ & $\begin{array}{l}\text { Vegetative state, unresponsive and } \\
\text { speechless }\end{array}$ \\
$\mathbf{2}$ & Death \\
$\mathbf{1}$ & \\
\hline
\end{tabular}

Data processing and analysis: After ensuring the consistency and completeness of the checklist, data were coded and entered into the computer using SPSS version 20, and descriptive analysis was done. Results were presented using frequency tables, graphs and percentages. Bivariate logistic regression analysis was done to determine crude statistical associations between independent variables and the dependent variable. Statistical significance was based on a p-value of $<0.05$ with confidence interval (CI) of $95 \%$.

\section{RESULTS}

Of the 195 charts reviewed and retrospectively evaluated, $137(70.3 \%)$ were of males, and $58(29.7 \%)$ (table 2 ) were of females patients with a $\mathrm{M}: \mathrm{F}$ ratio of $2.4: 1$. According to age categorization, $19(9.7 \%)$ of patients were between 15 and 34 years of age, $43(22.0 \%)$ were between 35 and 54 years, $110(56.4 \%)$ were between 55 and 74 years and $23(11.8 \%$ ) were older than 75 years of age (table 2). The age range was 19 years to 85 years old with the mean age of 57.63 .

Table 2: Socio-demographic Profile of the Patients

\begin{tabular}{lll}
\hline Variable & Category & Frequency (\%) \\
\hline \multirow{3}{*}{ Sex } & Male & $137(70.3)$ \\
& Female & $58(29.7)$ \\
Age & $15-34$ & $19(9.7)$ \\
& $35-54$ & $43(22.0)$ \\
& $55-74$ & $110(56.4)$ \\
& $\geq 75$ & $23(11.8)$ \\
\hline
\end{tabular}

The most common presenting symptom was headache which was present in $75.9 \%$ of the patients. Other presenting symptoms included extremity weakness in $133(68.2 \%)$, sphincter dysfunction in $59(30.2 \%)$, change in mentation in $50(25.6 \%)$, and memory loss in $14(7.2 \%)$ of the patients (table 3 ). History of head trauma prior to presentation was documented in 124 (63.6\%) patients, Seventy one $(36.3 \%)$ of the patients in this study had no obvious history of head trauma (table $3)$. 
Table 3: Clinical Presentation of the Patients

\begin{tabular}{ll}
\hline Variable & Frequency (\%) \\
\hline Presenting Symptoms & $148(75.9)$ \\
Headache & $133(68.2)$ \\
Extremity Weakness & $59(30.2)$ \\
Sphincter Dysfunction & $50(25.6)$ \\
Change in Mentation & $14(7.2)$ \\
Memory Loss & $124(63.6)$ \\
History of Head Trauma & $71(36.3)$ \\
Yes & \\
No & \\
\hline
\end{tabular}

There was statistically significant strong association of male sex with incidence of trauma induced CSDH. Of the 137 male patients in this study, $96(70.0 \%)$ had a positive history of head injury prior to presentation. In contrast, only $48.3 \%(28 / 58)$ of the female patients had history of head injury. Men had 3 times higher risk of developing traumatic CSDH than women with P-value of 0.004and 95\% CI $(4.90$ - 13.69).

All of our patients had at least one imaging study of the head with either CT scan or MRI. Based on the imaging findings, $80(41.0 \%)$ patients had a left side hematoma, 67(34.4\%) of patients had a right side and $48(24.6 \%)$ patients had bilateral CSDH (Figure 1). All patients were treated with a single burr hole evacuation under local anesthesia and postoperative subdural closed system drainage. The hospital stay of the patients ranged from one day, for a patient who died on the same day of the surgery, to 32 days, for one of our patients with associated sever cardiac illness with HAP on ICU management before the surgical procedure for $\mathrm{CSDH}$, making the mean hospital stay $3.68 \pm 2.6$ days.

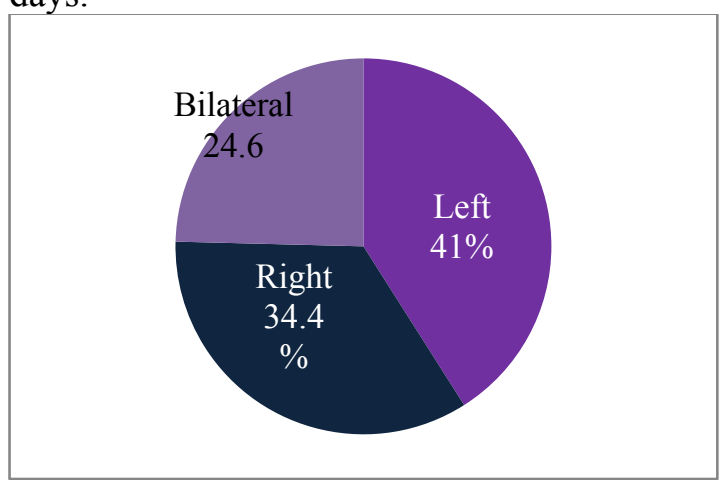

Figure 1: Site of Chronic Subdural Hematoma in Percent (\%)
The postoperative outcome of our patients was assessed using the Glasgow Outcome Score at the last postoperative follow-up. Ninety five point nine percent of our patients reported a good recovery at the end of the fourth month postoperative follow-up: $167(85.6 \%)$ reported good recovery with resumption of normal life (GOS 5) while 20(10.3\%) reported moderate disability (GOS 4). Four patients had persistent (GOS 3), and there were no patients with GOS of 2. Death occurred in four $(2.0 \%)$ of our patients (Table 4).

Table 4: Postoperative Outcome

\begin{tabular}{lll}
\hline $\begin{array}{l}\text { Glasgow } \\
\text { Outcome Score }\end{array}$ & Frequency & Percent \\
\hline 5 & 167 & 85.6 \\
4 & 20 & 10.3 \\
3 & 4 & 2.0 \\
2 & 0 & 0 \\
1 & 4 & 2.0 \\
\hline
\end{tabular}

Patients with traumatic CSDH had a better postoperative outcome, GOS 4 and 5, than those with non-traumatic CSDH. Furthermore, death was more common in patients with non-traumatic CSDH: 3 of the 4 deaths in our study occurred in patients with no obvious history of head injury. Therefore, there was a statistically significant association between history of head trauma and postoperative outcome, with $\mathrm{P}$ - value of 0.028 and $95 \%$ CI of $(1.113-25.64)$.

Hundred percent of our patients without history of extremity weakness had good recovery (GOS 4 and 5) whereas $94 \%$ of the patients with preoperative extremity weakness reported a postoperative GOS of 4 and 5. In addition, all the four deaths in our study occurred in patients with extremity weakness, showing statistically significant association between presence of preoperative extremity weakness and postoperative outcome with P-value of 0.042 and 95\% CI (1.02 1.11).

There was no statistically significant association between postoperative outcome with sex and age of patients, and other presenting symptoms such as change in mentation, sphincter dysfunction or memory disturbance. There was also no statistically significant association between recollection with age, sex or mode of presentation.

DOI: http://dx.doi.org/10.4314/ejhs.v30i1.13 
Thirteen $(6.6 \%)$ patients were operated twice for recollection, and the hematoma was evacuated through the same burr hole. There was no second time recollection, and all of these patients were discharged with improvement. Other postoperative complications such as intracerebral bleeding, tension pneumocephalus, postoperative seizures or infectious complication were not observed in our study.

\section{DISCUSSION}

Chronic subdural hematoma is a commonly encountered neurosurgical condition. The surgical management of this condition can safely be undertaken by neurosurgeons, general surgeons as well as residents in these disciplines. A study from Kenya showed that operative management of CSDH can be safely performed in rural facilities by general surgeons familiar with the procedure and with the institutional resources (15). Describing the safest, easy and effective procedure is very important particularly for developing countries, like Ethiopia, where CSDH is operated by neurosurgeons or general surgeons at primary level hospitals where the facility of such health institutions may not allow for sophisticated invasive surgeries for elderly patients.

In this study, CSDH was found to be more common in men than in women. Of the 195 patients operated, $137(70.3 \%)$ were male, and 58(29.7\%) were female patients with M:F ratio of 2.4:1. Similarly, a male predominance was found in Shizuoka, Japan (71.8\%) (8), Peshawar, Pakistan (80.0\%) (9), Cotonou, Benin (83.65\%) (10) and Accra, Ghana (92.6\%) (11). The mean age at presentation in this study was 57.63 , with $133(68.2 \%)$ of the patients were above 55 years of age. CSDH occurs predominantly in the elderly (35,7-11), and our study also showed a similar result.

The most common presenting symptom was headache followed by extremity weakness, sphincter dysfunction, change in mentation, and memory loss. History of head trauma prior to presentation was documented in $63.6 \%$ patients, and similar results have been reported in other studies $(2,9,10,11)$. Forty one percent of the patients had a left side hematoma, and $34.4 \%$ had right sided hematoma. Bilateral CSDH was found in $24.6 \%$ patients. Left sided hematoma was also found more common in a study from Japan, $52.0 \%$, and bilateral hematoma was found in $17.6 \%$ (8). In contrast, the study from Accra showed a predominance of right sided hematoma accounting for $58.3 \%$ with bilateral CSDH occurring in $11.5 \%$ of patients.

All of our patients were operated with a single burr hole evacuation with intraoperative irrigation and postoperative subdural closed system drainage by a single neurosurgeon. Burr hole craniostomy is the most effective procedure and has the best cure to complication ratio $(12,13)$. The mean hospital stay in our study was $3.68 \pm 2.6$ days which is almost similar to that reported in Rochester, USA, where it was $4.3 \pm 2.2$ for the same procedure (13). One hundred eighty seven (187) $(95.9 \%)$ of our patients had good recovery, GOS 4 and GOS 5, at the time of their last postoperative visit, and there were four $(2.0 \%)$ deaths. This was similar to the study done in Accra, Ghana, where $93.8 \%$ had good recovery with mortality of $2.0 \%$ (11).

The proposed criteria for the diagnosis of early recurrent CSDH are the return of symptoms or reaccumulation of the hematoma after a surgery within 3 months regardless of the location, amount or repeated operations (14). Recurrence occurred in 13(6.6\%) patients. The reported rates of recurrence include $1 \%$, $6.7 \%$, and $9.8 \%$ in Ghana, Pakistan and Japan, respectively $(8,9,11)$. All patients with recurrent subdural hematoma were treated with evacuation of the hematoma through the same burr hole. Burr hole craniostomy is the most effective procedure in treating recurrent hematoma with craniotomy reserved as the treatment of last choice (12). We found no association between recurrence with age, sex, etiology or mode of presentation. Postoperative complications such as intracerebral bleeding, tension pneumocephalus, seizures or infectious complications were not observed in this study. There were four postoperative deaths. Two of these deaths were of patients with Glasgow Coma Scale of 5-6 with associated aspiration pneumonia: one on the same day of the surgery and the other two days after the surgery. The third death encountered developed massive heamorrhage from multiple mucocutaneous sites on the $8^{\text {th }}$ postoperative day. The last documented death was of an 80 year old man who died on the $11^{\text {th }}$ postoperative day from cardiac arrest while he was prepared for discharge from the hospital.

In this study, postoperative outcome was worse in patients with non-traumatic CSDH and those with preoperative extremity weakness. There was no statistically significant association between postoperative outcome with sex and age of patients, and other presenting symptoms such as change in

DOI: http://dx.doi.org/10.4314/ejhs.v30i1.13 
mentation, sphincter dysfunction or memory disturbance.

The study had limitations as it was a single institutional study and the findings do not represent the whole population of the country. Duration of illness was not clearly documented, and its association with postoperative outcome was not evaluated in this study. Because of the retrospective nature of this study, it also had limitations of being unable to analyze the preoperative Glasgow Coma Scale of the whole patients and also failed to see any post- complications from post-operative supine position of patients for 48 hours.

We conclude that a single burr-hole craniostomy and closed system subdural drainage of CSDH under local anesthesia with occasional sedation for uncooperative patients due to altered mentation is an easy, safe and effective technique for the treatment of $\mathrm{CSDH}$ which can be performed in primary level hospital. We recommend that all surgeons in our country should be familiarized with the single burr hole craniostomy technique as it is safe, easy, and life saving procedure for the treatment of CSDH.

\section{ACKNOWLEDGEMENTS}

We would like to acknowledge the assistance of Teklehaymanot General Hospital in undertaking this research. We also like to thank the members of the record room staff at the hospital for their valuable cooperation. Last but not least, we would like to express our gratitude and utmost appreciation to the ward and operating theater's nurses, anesthetists and anesthesiologists, surgeons and supporting staff who are working tirelessly in the management of our patients.

\section{REFERENCES}

1. Yoji Tanaka and KikuoOhno. Chronic Subdural Hematoma- An Up-to-Date Concept, Review. J Med Dent Sci, 2013; 48:55-61.

2. Dipu Bhuyan, Pradipta Ray Choudhury, MrinalBhuyan. Burr-hole irrigation of Hematoma Cavity with and without Drainage in the Treatment of Chronic Subdural Hematoma: A Comparative Study. International Journal of Scientific Study, 2016; 3(12):53-57.

3. Ajaya KumerA, Grace Varghese, Litta Thomas. Series Study of Subacute and Chronic subdural hematoma. J Neurol Stroke 2016; 5(2):00168.

4. Mark S Greenberg Chronic shbdural hematoma: hand book of neurosurgery. ( $8^{\text {th }}$ edn), p. $899-903$.

5. Joon Kook Lee, Jong Hun Choi, Chang Hyun Kim, Ho Kook Lee, Jae Gon Moon. Chronic Subdural
Hematomas: A Comparative Study of Three Types of Operative Procedures. J Korean NeurosurgSoc, 2009; 46: $210-214$.

6. Aslan Guzel, Sedat Kaya, UmitOzkan, M UfukAluclu, Adan Ceviz, Deniz Belen. Surgical treatment of chronic subdural hematoma under monitored anesthesia care. Swiss Med Wkly, 2008; 138(27-28): 398-403.

7. Weiming Liu, M.D., Nicolaas A. Bakker, M.D., PH.D., and Rob J. M. Groen, M.D., PH.D.; Chronic Subdural Hematoma: a systematic review and metaanalysis of surgical procedures. J Neurosurg, 2014;121:665-673.

8. Kentaro MORI and Minoru MAEDA. Surgical Treatment of Chronic Subdural Hematoma in 500 consecutive cases: clinical Characteristics, surgical Outcome, Complications, and Recurrence Rate. Neurol Med Chir(Tokyo), 2001;41:371-381.

9. RiazurRehman, AzmatullahKhattak, Farooq Azam, WaqarAlam, Anayatullah; Outcome of Chronic subdural Hematoma patients treated by Two Burr Holes Method. Gomal Journal of Medical Sciences, 2010:8 (2):161- 165.

10. LuphinHode, Kisito Quenum, Holden O. Fatigba LionelleFanou, Henderson G. S. Lansdale. Treatment and Outcome of Chronic Subdural Hematoma in SubSaharan Africa and the country of Benin. Iranian Journal of Neurosurgery, 2015; 1(2):31-35.

11. T. K. Dakurah, M. Iddrissu, G. Wepeba and I. Nuamah. Chronic Subdural Hematoma: Review of 96 cases attending the Korle Bu Teaching hospital, Accra. WAJM, 2005; 24(4):283-286.

12. R. Weigel, P Schmiedek, J K Krauss. Outcome of contemporary surgery for chronic subdural haematoma: evidence based review. J NeurolNeursurg Psychiatry, 2003; 74:937-943.

13. Vanessa Milano, M.D., Howard Silberstien, M. D. Surgical Management of Chronic Subdural Hematomas: Comparison of Outcomes and Efficacy. University of Rochester Medical Center, Department of Neurosurgery.

14. Hyuck-Jin Oh, M.D., Kyeong-Seok Lee, M.D., JaeJun Shim, M.D., Seok-Mann Yoon, M.D., Il-Gyu Yun, M.D., Hack-Gun Bae, M.D.Postoperative Course and Recurrence of Chronic Subdural Hematoma. J Korean NeurosurgSoc, 2010; 48: 518-523.

15. John K. Kanyi, Timothy V. Ogada, Mark J. Oloo, Robert K. Parker. Burr-hole Craniostomy for Chronic Subdural Hematomas by General Surgeons in Rural Kenya. World Journal of Surgery January, 2018; 42(1): 40

DOI: http://dx.doi.org/10.4314/ejhs.v30i1.13 